\title{
Variable accretion and outflow in young brown dwarfs
}

\author{
Alexander Scholz and Ray Jayawardhana \\ Department of Astronomy \& Astrophysics, University of Toronto, 60 St. George Street, \\ Toronto, Ontario M5S3H8, Canada \\ aleks@astro.utoronto.ca
}

\begin{abstract}
We report on the first dedicated monitoring campaign of spectroscopic variability in young brown dwarfs. High-resolution optical spectra of six targets in nearby star-forming regions were obtained over 11 nights between 2005 JanuaryMarch on the Magellan 6.5m telescope. We find significant variability in $\mathrm{H} \alpha$ and a number of other emission lines related to accretion and outflow processes on a variety of timescales ranging from hours to weeks to years. The most dramatic changes are seen for 2MASS J1207334-393254 (2M1207), 2MASS J110132057718249 (2M1101) and ChaI-ISO217. We observe possible accretion rate changes by about an order of magnitude in two of these objects, over timescales of weeks (2M1207) or hours (2M1101). The accretion 'burst' seen in 2M1101 could be due to a 'clumpy' flow. We also see indications for changes in the outflow rate in at least three objects. In one case (ISO217), there appears to be a $\sim 1$-hour time lag between outflow and accretion variations, consistent with a scenario in which the wind originates from the inner disk edge. For some objects there is evidence for emission line variability induced by rotation. Our variability study supports a close to edge-on inclination for the brown dwarf LS-RCrA 1. The fact that all targets in our sample show variations in accretion and/or outflow indicators suggests that studies of young brown dwarf properties should be based either on large samples or time series. As an example, we demonstrate that the large scatter in the recently found accretion rate vs. mass relationship can be explained primarily with variability. The observed profile variations imply asymmetric accretion flows in brown dwarfs, which, in turn, is evidence for magnetic funneling by large-scale fields. We show that accreting sub-stellar objects may harbor magnetic fields with $\sim \mathrm{kG}$ strength.
\end{abstract}

Subject headings: stars: formation, low-mass, brown dwarfs, winds, outflows line: profiles, formation - accretion, accretion disks 


\section{Introduction}

Brown dwarfs (BDs) are objects with masses intermediate between stars and planets. From a variety of observational studies, there is now clear evidence that many BDs with ages $<10$ Myr share properties with solar-mass classical T Tauri stars (CTTS), which are in a phase of active accretion. Young BDs have been found to harbour circum-sub-stellar disks, as indicated by near- and mid-infrared fluxes significantly higher than the photospheric fluxes from the object itself. Their spectral energy distribution in the infrared and in the sub-millimeter regime is nicely reproduced by disk models with either flat or flared disk geometry (e.g., Natta et al. 2002; Jayawardhana et al. 2003; Pascucci et al. 2003; Mohanty et al. 2004). Moreover, a large fraction of young BDs show the typical emission line spectrum of $\mathrm{T}$ Tauri stars, with broad, asymmetric $\mathrm{H} \alpha$ lines, and additional emission lines like HeI and OI, which is direct evidence for ongoing accretion (e.g., Jayawardhana, Mohanty, \& Basri 2002, 2003; White \& Basri 2003; Muzerolle et al. 2003; Natta et al. 2004; Mohanty, Jayawardhana, \& Basri 2005). The accretion process is also believed to produce the largeamplitude, partly irregular photometric variability observed in young BDs (Scholz \& Eislöffel 2004, 2005). Finally, for some substellar objects outflows have been detected as forbidden line emission in [SII] and [OI] (Fernández \& Comerón 2001; Barrado y Navascués, Mohanty, \& Jayawardhana 2004; Barrado y Navascués \& Jayawardhana 2004; Whelan et al. 2005). Thus, it is quite clear that many young BDs undergo an accretion phase similar to more massive objects.

The general picture of accretion on T Tauri stars is the magnetospheric accretion model. In this view, the accretion is funnelled by a large-scale magnetic field structure, which truncates the disk at a certain inner radius. The gas flows with nearly free-fall velocity from the disk to the star following the magnetic field lines and finally forms an accretion shock and hot spots near the atmosphere of the accreting object (e.g., Koenigl 1991; Shu et al. 1994; Hartmann, Hewett, \& Calvet 1994). Many recent studies provide ample support for this idea of magnetically channelled accretion, and there is growing evidence that this scenario also applies to substellar objects.

Perhaps the most detailed view on the accretion process can be obtained by highresolution optical spectroscopy. By analysing the emission line fluxes and profiles, it is possible to constrain the accretion parameters such as the infall rate or the temperature in the flow. Accreting objects, however, show a puzzling variety of emission line profiles, from nearly symmetric and double-peaked to classical or inverse P Cygni type features (e.g. Fernández et al. 1995; Reipurth, Pedrosa, \& Lago 1996). Part of this diversity may be explained by different accretion flow geometries in individual objects. But we also have to take into account that we see the star-disk system in different projections. In the two 
extreme cases, the line of sight can point directly to the edge of the disk (edge-on view) or to the pole (face-on view). As a consequence, for different objects the received flux comes from different parts of the disk and the object, whereas other parts may be obscured.

On the other hand, it has long been known that $\mathrm{T}$ Tauri stars are variable on timescales ranging from a few hours to years (e.g. Herbst et al. 1994; Johns \& Basri 1995; Bouvier et al. 1999, 2003). Hot spots formed by the accretion flow co-rotate with the object, and might therefore not always be visible to the observer. Moreover, the rotational axis can be inclined with respect to the magnetic axis, resulting in a non-axisymmetric flow (Romanova et al. 2003). Simulations show that the magnetospheric accretion itself is unlikely to be stable over long timescales; reasons for instabilities include recurrent opening of the magnetic field lines (e.g. Uzdensky 2002; Matt \& Pudritz 2005) and clumpy accretion (Mitskevich, Natta, \& Grinin 1993). All these processes can lead to variability and, in particular, to variations in the emission line profiles. Summarising, rotation, accretion instabilities, geometry effects, and differences between individual objects are mainly responsible for the different types of emission line profiles observed for accreting objects.

From this reasoning it becomes clear that variability studies, in particular spectroscopic monitoring, can be used to obtain a close-up view on the accretion behaviour of individual objects, because they help to disentangle the different effects which influence the line profiles. Indeed, from intensive monitoring campaigns for selected CTTS, it has been possible to derive a detailed description of the accretion process for some objects (e.g. Johns-Krull \& Basri 1997; Alencar \& Batalha 2002; Bouvier et al. 2003). At a minimum, variability studies provide information about the range of accretion parameters on a given object, allowing us an unbiased comparison between different objects. Thus, variability studies for individual targets are a valuable complement to the statistical analysis of large object samples.

Therefore, we have carried out the first spectroscopic monitoring campaigns of young, accreting brown dwarfs. We selected targets which show broad and asymmetric $\mathrm{H} \alpha$ profile in previous studies, indicative of infalling and/or outflowing material (see Sec. 2.2). In addition, we aimed to cover a large variety of emission line profiles and thus accretion properties. Our final sample of six objects is given in Table 1, which additionally contains aliases for all targets. In the remainder of the paper, we will use these short names. For all six objects, the membership in nearby star-forming regions has been confirmed spectroscopically, and all have spectral types later than M6, indicating that they are either very close to or below the substellar limit (see Luhman et al. 2003). For a more precise mass estimate for these objects, a better calibration of the stellar evolutionary tracks for young objects is required (see Baraffe et al. 2002). However, since crossing the substellar limit is not expected to alter the accretion process in any way, we will conveniently call all targets 'brown dwarfs' in the 
rest of the paper, although not all of them may be bona fide substellar objects.

First results from this study have already been published in Scholz, Jayawardhana, \& Brandeker (2005) (hereafter SJB05), where we report on dramatic changes in the emission lines of the BD 2M1207 (see Table 1). In this paper, we give a consistent description of the emission line variability for six targets, including 2M1207. In Sec. 2 we present our observations and data reduction. Subsequently, we give a phenomenological description of the emission line spectra, including an analysis of the profile of the most prominent feature, the $\mathrm{H} \alpha$ line (Sec. 3), and a survey of other features seen in our data (Sec. 4). This information will then be used to constrain the accretion behaviour of our targets (Sect. 5) and brown dwarfs in general (Sec. 6). Finally, we give our conclusions in Sec. 7.

\section{Observations and data analysis}

\subsection{Observation and basic reduction}

All spectra for this study have been taken with the MIKE spectrograph at the MagellanClay $6.5 \mathrm{~m}$ telescope on Las Campanas. MIKE is a double echelle instrument, consisting of blue and red arms, providing coverage from 3400 to $5000 \AA$ and 4900 to $9300 \AA$, respectively, in our configuration. We obtained most of the time series spectra for our six targets in an observing run from 17 to 19th of March 2005. Except for $\mathrm{ChaH} \alpha 1$, at least one additional spectrum per target was taken in a second observing run at the end of March. Three extra spectra for 2M1207 were obtained in a preceding campaign in January-February 2005. In total, the variability study is based on 13 spectra for 2M1207, seven for 2M1101, five for $\rho$ Oph-32, ISO217, ChaH $\alpha-1$, and four for LS-RCrA 1 (see Table 2). In the main observing run, we used on-chip binning of $2 \times 2$ pixels in combination with slit sizes of 1 ". 0 or 0 ".7, resulting in a resolution of $R \sim 20000$. All exposures were split into three single spectra to avoid excessive contamination by cosmic rays. The two auxiliary runs had the primary goal to measure high-precision radial velocities, therefore we aimed for the highest possible resolution. For that reason, spectra from these runs were taken with a 0"35 slit in combination with either no or $2 \times 2$ binning. Therefore, the data from our primary run have in general a higher signal-to-noise ratio (hereafter $\mathrm{s} / \mathrm{n}$ ), but lower resolution. The exposure times for our targets were between 15 and $60 \mathrm{~min}$. In Table 2 we list for all spectra the observing times, configurations, and exposure times. In all runs, we additionally observed a spectrophotometric standard star for order definition and (relative) flux calibration.

The basic reduction of all spectra was done using standard routines in the doecslit 
package within IRAF $^{1}$. The procedure includes overscan subtraction, flatfield correction, sky background subtraction, order extraction, and blaze correction using the standard star spectrum. The wavelength calibration was carried out using Thorium-Argon spectra; we obtained a typical precision of a few $m \AA$ for the wavelength solution. Cosmic rays were removed by applying a small-scale median filter to each order, comparing the filtered spectrum with the original data, and replacing all outliers with the median value. In cases where we split the total exposure time to several spectra, these single exposures were co-added after the extraction.

\subsection{Line measurements}

Our spectra show a variety of emission lines, which are believed to form either by accretion, outflows/winds, or magnetic activity. We selected a set of features, which should allow us to disentangle the effects of these three processes, and measured equivalent widths (hereafter EW) for all of them.

The dominant emission line in all spectra is the $\mathrm{H} \alpha$ feature at $6562.8 \AA$, which has its origin in the chromosphere and/or in the accretion flow. To decide whether an object is accreting or not, we take advantage of the fact that we expect the material in the accretion flow to be moving close to free-fall velocity, which is $\sim 150 \mathrm{kms}^{-1}$, whereas the velocity for chromospheric emission is usually significantly smaller. Based on these considerations, White \& Basri (2003) and Jayawardhana, Mohanty, \& Basri (2003) introduced the $10 \%$ width of the full peak height (hereafter $10 \%$ width) as a useful measurement to discriminate accretors and non-accretors. Jayawardhana, Mohanty, \& Basri (2003) suggested a threshold of $\sim 200 \mathrm{kms}^{-1}$ for substellar objects, and Mohanty, Jayawardhana, \& Basri (2005) demonstrate that this is a reasonable value to separate accreting and merely chromospherically active objects. It turned out that the $10 \%$ widths are indeed nicely correlated with the accretion rates derived from conventional veiling analysis (Natta et al. 2004). It can therefore be used to obtain an accretion rate estimate from the emission line profile, which is particularly useful for BDs, where the continuum is faint. It should be noted, however, that an extremely active and fast rotating object might be able to produce a $10 \%$ width of about $200 \mathrm{kms}^{-1}$ or more. From our targets, however, none is known to be an exceptional fast rotator. The $v \sin i$ values derived so far (see Table 1) indicate rotational velocities $<20 \mathrm{kms}^{-1}$.

The profile of $\mathrm{H} \alpha$ can be used as an additional indicator for ongoing accretion: Chro-

\footnotetext{
${ }^{1}$ IRAF is distributed by National Optical Astronomy Observatories, which is operated by the Association of Universities for Research in Astronomy, Inc., under contract with the National Science Foundation.
} 
mospheric $\mathrm{H} \alpha$ profiles are in most cases more or less symmetric, and often a small central dip due to self-absorption in the chromosphere is seen. On the other hand, accreting objects usually exhibit strongly asymmetric profiles, with broad wings and either red- or blue-shifted absorption features, indicative of high-velocity infalling or outflowing material. In the following, we will therefore use the $10 \%$ width and the shape of the profile to distinguish between non-accretors and accretors.

All our targets show emission in the higher Balmer lines $\mathrm{H} \beta$ and $\mathrm{H} \gamma$, which require higher temperature regions in comparison with $\mathrm{H} \alpha$. Again, the width of the lines can be used to discriminate accretors and non-accretors. Additionally, many spectra show HeI, most prominently at 5875 and $6678 \AA$, OI at $8446 \AA$, and the CaII triplet in emission. If detected, we measured EW for these lines. The HeI5875 feature is known to be in absorption in many chromospherically active stars, but it can also appear in emission during flares in active M dwarfs (Saar et al. 1997, and references therein). Although young BDs are known to show very few flares (see Scholz \& Eislöffel 2005), we cannot exclude that the HeI5875 emission has its origin in the chromosphere. HeI6678, OI8446, and the CaII triplet, however, appear preferentially in accretors, as argued by Mohanty, Jayawardhana, \& Basri (2005). Therefore we use these lines as complementary information to distinguish between accretors and non-accretors. Particularly the HeI6678 EW can be used as a strong discriminator between accretors and non-accretors: Gizis, Reid, \& Hawley (2002) observed 676 M dwarfs, most of them active, in the solar neighbourhood, and although many of them have HeI6678 in emission, none has an EW larger than $0.25 \AA$. For our spectra, this is lower than the detection limit, i.e. if we detect HeI6678 in emission, it is very likely caused by accretion.

Similarly to $\mathrm{H} \alpha$, many emission lines of accreting objects can be formed in infalling as well as in the chromosphere or in outflowing material, as has been shown at least for HeI (Beristain, Edwards, \& Kwan 2001) and CaII (see Fernández \& Comerón 2001). The line ratio in the CaII triplet, however, gives a first indication of whether the flux comes mainly from infall or outflow: For low-density outflowing material, we expect line ratios of 1:9:5, as it has been observed for Herbig Haro objects (Reipurth et al. 1986). On the other hand, line ratios close to 1:1:1 imply saturation and optically thick CaII emission, as argued by Graham \& Heyer (1988), and is therefore evidence for an accretion-related origin.

Some objects show clear evidence for forbidden line emission, particularly in [SII] $(6730 \AA)$ and [OI] (6300 $\AA$ ). If possible, we measured EW for these lines as well. Forbidden line emission is a clear indication for mass outflow, since these lines can only form in low-density regions, and not in the accretion shock (see Hirth, Mundt, \& Solf 1997). They can therefore also be used to decide whether the permitted emission lines are mainly formed by outflow or infall. 
For all line measurements, the spectra were shifted to the local stellar rest frame. We used literature radial velocities for 2M1207, ChaH $\alpha 1$, and LS-RCrA 1 (see Table 1). The uncertainties of these measurements are at most $3 \mathrm{kms}^{-1}$. For the remaining three targets, there is no previous radial velocity measurement. Using the Li6708 absorption feature, we are able to derive an estimate of $0 \pm 3 \mathrm{kms}^{-1}$ for $\rho \mathrm{Oph}-32$, which is roughly consistent with the velocity of the cloud and of young stars in $\rho$ Oph (see Doppmann et al. 2005). 2M1101 and ISO217 belong to the ChaI star forming region, where the brown dwarfs have been found to have average radial velocities of $\sim 15 \mathrm{kms}^{-1}$ with only a small dispersion of $2-3 \mathrm{kms}^{-1}$ (Joergens \& Guenther 2001; Joergens 2005). For these two targets, we therefore adopt the ChaI average value as radial velocity. Since the radial velocity error is the main source of uncertainty in velocity, we estimate our error for all velocity values to be $\sim 3 \mathrm{kms}^{-1}$, still lower than our spectral resolution.

\subsection{Continuum analysis}

Our general observing strategy was to keep the exposure time as short as possible, to be able to obtain more than one spectrum per night for each of our target. As a consequence, the $\mathrm{s} / \mathrm{n}$ in the continuum is low, particularly for $\lambda<7000 \AA$, deterring us from a detailed analysis of the photospheric lines for our objects. Many important emission lines are in regions with faint continuum, e.g. the complete Hydrogen Balmer series, and we have to make sure that the continuum emission is dominated by the flux from the target, and not significantly contaminated by imperfect background or flatfield correction. We compared the averaged spectral energy distribution (SED) in our data with model spectra from Allard, Hauschildt, \& Schwenke (2000), selected to match effective temperature and gravity of our targets. Both the observed and the theoretical SED were normalised to the flux at $8600 \AA$, where we safely detect the continuum in all our spectra with $\mathrm{s} / \mathrm{n}>10$, and the ratio between observed and modeled spectrum was computed. This ratio is expected to scatter around unity as long as the observed continuum is determined predominantly by the photospheric flux from the target. For all our targets we find good agreement between observed and model SED for $\lambda>4800 \AA$. As expected, the limit for a reliable continuum detection is a function of $\mathrm{s} / \mathrm{n}$. We conclude that all line measurements down to the $\mathrm{H} \beta$ line at $4861 \AA$ are reliable.

Since all our targets are known to accrete, they might show excess continuum emission due to the so-called veiling, which is produced in the accretion funnel and 'fills' the photospheric absorption lines. Strong veiling is often seen in accreting $\mathrm{T}$ Tauri stars, and is known to correlate with the accretion rates (e.g., Basri \& Batalha 1990). Since the veiling is superimposed on the photospheric flux, it may prevent a reliable continuum measurement 
and thus influence the equivalent widths. Recent studies, however, have shown that the accretion rates steadily decrease with the mass of the central object. As a consequence, the average accretion rates of brown dwarfs are in the range of $10^{-11} M_{\odot} \mathrm{yr}^{-1}$ (Muzerolle et al. 2003; Natta et al. 2004; Mohanty, Jayawardhana, \& Basri 2005). Even in our sample of strongly accreting brown dwarfs, the mean accretion rate is probably only $10^{-10} M_{\odot} \mathrm{yr}^{-1}$. At these low accretion levels the veiling is safely below $10 \%$ at least for $\lambda>5500 \AA$ (White \& Basri 2003; Muzerolle et al. 2003), and thus negligible (and not detectable) in the red part of our spectra.

Veiling is a strong function of wavelength and in most cases becomes stronger in the blue spectral range. At $5000 \AA$ it can be twice as strong as at $7000 \AA$, as shown for CTTS (Alencar \& Batalha 2002; Basri \& Batalha 1990). Discrepancies between photospheric and observed SED for $\lambda<4800 \AA$, as we have detected in some cases, might therefore be caused by veiling, although this is unlikely given the fact that these discrepancies are strongest in spectra with very poor $\mathrm{s} / \mathrm{n}$, which clearly points to an instrumental effect. Therefore, our line measurements down to $\mathrm{H} \beta$ are probably not significantly affected by veiling.

\section{3. $\mathbf{H} \alpha$ variability}

The dominant emission feature for all targets is the $\mathrm{H} \alpha$ line at $6562.8 \AA$. Our highresolution spectra allow us a detailed analysis of the line profile. In Fig. 1 we present the average $\mathrm{H} \alpha$ profile $\bar{I}(v)$ for all six targets, calculated over the complete time series. All single spectra were normalised to the continuum level before the averaging. In addition, we derived the normalised variance profile $\sigma_{N}^{2}(v)$ following Johns \& Basri (1995) by calculating the variance in in each velocity bin and normalising by $\bar{I}(v)$. This allows us to quantify the variability as a function of velocity. Both profiles are determined by averaging over a moving velocity window with a width of $10 \mathrm{kms}^{-1}$; the stepsize is $1 \mathrm{kms}^{-1}$.

To characterise the total amount of variability, Johns \& Basri (1995) used the EW of the variance profile, i.e. the integral over $\sigma_{N}^{2}(v)$. If we use this criterion, three of our targets are highly variable (2M1207, 2M1101, ISO217), but for the remaining three the $\mathrm{H} \alpha$ profile is more or less constant. Using the EW variance as variability measurement implicitly assumes that the zero variability level is constant with velocity, which is usually the case for stars with strong continuum. In our spectra, however, the $\mathrm{s} / \mathrm{n}$ changes drastically with velocity; it is much higher in the core of $\mathrm{H} \alpha$ than in the wings and the continuum. As can be seen from Fig. 1, the normalised variance in the continuum $\left(\sigma_{N, 0}^{2}\right)$ is in the range of 0.2-0.5 for our targets, implying a $\mathrm{s} / \mathrm{n}$ of 1.5-2.5 in the continuum. Assuming Poisson noise, the expected $\sigma_{N}^{2}(v)$ without any variability (i.e. the zero variability level) can be estimated 
as $\sigma_{N}^{2}(v)=\sigma_{N, 0}^{2} \times(\sqrt{\bar{I}} / \bar{I})^{2}$. This function is shown with dashed lines in Fig. 1 . As expected, the continuum is non-variable (by definition), because all spectra were normalised to the continuum before averaging. Using this zero-variability level, it is now clear that all six targets show significant variability in the profile. As a better way to assess the total variability in $\mathrm{H} \alpha$ we define the $\mathrm{H} \alpha$ variability index ( $\mathrm{H} \alpha \mathrm{VI})$, calculated by integrating the difference between variance profile and zero variability level between -400 and $400 \mathrm{kms}^{-1}$. These values are given in the last column of Table 1.

Our targets show a large variety of $\mathrm{H} \alpha$ profiles, as apparent from the average profiles. The variance profiles exhibit in most cases a blue-shifted and a red-shifted peak at velocities between 50 and $150 \mathrm{kms}^{-1}$, sometimes a third central peak is visible. Three of our objects (2M1207, 2M1101, ISO217) show strong variability in this line with $\mathrm{H} \alpha \mathrm{VI}>25$, whereas the remaining three targets are less variable. The variance profiles are in most cases quite different from the average profiles, demonstrating that the line variability is not only due to changes in the underlying continuum, because in this case the two profiles would be very similar (see Johns \& Basri 1995; Alencar \& Batalha 2002).

2M1207 shows clear indications for an asymmetry on the red side, most likely caused by red-shifted absorption features in parts of the time series (see SJB05). The variance profile clearly has three peaks, where most of the variability is produced in the blue wing of the line. 2M1101 has only a minor asymmetry in the profile with a hint of a red-shifted second peak. This is surprising, given the fact that the only available literature spectrum for this object shows a $\mathrm{P}$ Cygni type $\mathrm{H} \alpha$ profile with very strong absorption in the blue wing (Muzerolle et al. 2005). In contrast to $2 \mathrm{M} 1207$, here the variability in $\mathrm{H} \alpha$ is mainly in the red part of the line. ISO217 has a double-peaked profile in all spectra, where the blue peak is significantly weaker than the red one. Both peaks also appear in the variance profile, but here they have about the same intensity. The remaining three targets have a single-peak profile, and the variance profiles do not show strong features. ChaH $\alpha 1$ and LS-RCrA 1 have a small central absorption dip, whereas $\rho$ Oph-32 has a slight asymmetry on the blue side, which may be due to a blue-shifted absorption feature.

In Figs. 2 and 3 we show the complete $\mathrm{H} \alpha$ time series for two strongly variable objects, 2M1101 and ISO217. The same plots for 2M1207 are already contained in SJB05. 2M1101 exhibits weak, symmetric $\mathrm{H} \alpha$ profiles in four spectra, but strongly enhanced emission in the remaining three. The object ISO217 has a strongly double-peaked line in all exposures, where both the total intensity and the relative strength of the two peaks changes significantly. In most cases, however, the red peak is stronger than the blue one.

As already discussed in Sec. 2.2, the $10 \%$ width can be used as a robust measurement of the accretion rate. Our time series show that the $10 \%$ width is much less sensitive to 
variability compared with the EW: Whereas the $\mathrm{H} \alpha \mathrm{EWs}$ change by at least a factor of two in the time series, the $10 \%$ widths are constant with $\pm 15 \%$ for four out of six targets. For two objects, however, the $10 \%$ widths change significantly: For 2M1207, we reported the strong variability in the $10 \%$ width already in SJB05. We found an increase of the linewidth by $32 \%$ between the January and the March data, i.e. on a timescale of six weeks. In the March data, however, the $10 \%$ width is variable only to a much smaller extent. Strong linewidth variations are also seen for 2M1101 (see Table. 2): The 10\% width roughly doubles between spectra 3 and 4 , which are seperated by only 8 hours. In the subsequent exposures, the linewidth gradually decreases. The strong increase coincides with the appearance of an asymmetric $\mathrm{H} \alpha$ profile (see above) and other accretion-related lines in the spectrum (see Sec. 4 ), and it is responsible for most of the variability observed for this target. We note that the third target which is highly variable in EW and profile, ISO217, shows only minor changes in $10 \%$ width.

Another way of quantifying the asymmetry is to measure the $10 \%$ width for blue and red side of the profile separately. For the three targets without notable asymmetry in the line profile (ChaH $\alpha 1, \rho$ Oph-32, LS-RCrA 1), the difference between 'red' and 'blue' 10\% width ranges from -20 to $20 \mathrm{kms}^{-1}$. The objects with asymmetric profiles and high variability in $\mathrm{H} \alpha$, however, show strong deviations between red and blue linewidths (see. Fig. 4). For 2M1207, the line is broader on the blue side in spectra with no clear second peak on the red side. As a consequence, the difference between red and blue linewidth varies over $\sim 60 \mathrm{kms}^{-1}$ on timescales of a few hours. For 2M1101 the datapoints are mostly negative with an average of $\sim-20 \mathrm{kms}^{-1}$, showing that the line is always broader in the red compared to the blue. This might be an indication for absorption in the blue part, which has been seen before for this object (Muzerolle et al. 2005). As already mentioned, there is no clear evidence for blue absorption from the profile shape alone. The third strongly variable target, ISO217, shows significant asymmetry only in the first spectra, with a difference between red and blue linewidth of $\sim-40 \mathrm{kms}^{-1}$.

Two targets (2M1207 and ISO217) exhibit, at least in some cases, a double-peaked structure in $\mathrm{H} \alpha$ that we interpret as a superposition of a broad emission and an absorption component. To verify this hypothesis, we decompose the line for all clearly double-peaked spectra by adding two Gaussians, one for the emission and a second one for the absorption component. This decomposition technique is a standard way to disentangle the effects of different components in line profiles (see, e.g., Johns-Krull \& Basri 1997; Alencar \& Batalha 2002). The feasability of the method is demonstrated in Fig. 5 for both objects.

For 2M1207, the best fit closely matches the blue peak and is in reasonably good agreement with the red peak. The line wings are more intense than predicted by the Gaussian, 
but in general the fit is acceptable. We are, however, not able to obtain a plausible fit if we use two emission features. In the three double-peaked profiles, both components are clearly red-shifted, where the emission is located at $\sim 22 \mathrm{kms}^{-1}$ and the absorption at $\sim 31 \mathrm{kms}^{-1}$. The linewidths, EWs and fluxes for the emission component are higher than those for the absorption component by a factor of $2-3$ in all three spectra.

The decomposition works similarly well for ISO217, where we are also able to reproduce the line wings with the fit. The broad emission profile and the absorption feature are both blue-shifted, but in contrast to the emission, which is constant in position within our uncertainties (average $\sim-8 \mathrm{kms}^{-1}$ ), the absorption feature clearly changes its position on timescales of days. It is located at $-4 \mathrm{kms}^{-1}$ at $18 \mathrm{th}$ March, at $-15 \mathrm{kms}^{-1}$ at 19 th March, and at $-26 \mathrm{kms}^{-1}$ at 26 th March. The EW and flux of the emission is higher by a factor 3-4 than the values for the absorption.

\section{Emission line survey}

In the following we give a phenomenological description of the emission line spectrum for each target seperately. In addition, we check for correlations in the EWs of different lines. In general, all spectra show a strong $\mathrm{H} \alpha$ line (see Sec. 3), and most of them exhibit other emission lines indicative of either accretion or outflows or both. All objects show variability in the emission lines, with the most dramatic changes observed for 2M1207, 2M1101, and ISO217 as is also the case with $\mathrm{H} \alpha$ emission in Sec. 3.

2M1207: As already discussed in SJB05, the spectrum of 2M1207 is dominated by a variety of accretion related emission lines. It shows strong Balmer lines in some cases down to H10 at $3800 \AA$, both HeI lines, the CaII triplet, and OI. We do not see evidence for forbidden line emission in $[\mathrm{SII}]$ or $[\mathrm{OI}]$ in our spectra. The intensity of the $\mathrm{H}$ Balmer and HeI lines is strongly variable, the EWs change by a factor of 5-10. If we separate January and March data, however, the variations in $\mathrm{H} \alpha, \mathrm{H} \beta$, and HeI5875 are significantly smaller, indicating that most of the line variability is produced on timescales of a few weeks rather than days.

Only HeI6678 is not strongly enhanced in the March spectra. The CaII triplet lines are weakly detected in March, with more or less constant EWs of $\sim 0.6 \AA$, and all three lines show roughly the same intensity. Similarly, OI is only present in the March spectra. The EW of all Balmer lines are correlated, where the best correlations are seen between neighbouring lines in the series, e.g. for $\mathrm{H} \alpha$ vs. $\mathrm{H} \beta$ and $\mathrm{H} \beta$ vs. $\mathrm{H} \gamma$. HeI5875 is correlated with all Balmer lines, except for two datapoints in March, where this line shows an enhancement. This 'burst' is also seen in the high-energy Balmer lines, but at a weaker level. Our March EW in $\mathrm{H} \alpha$ are comparable to the literature values from Gizis $(2002$, EW of $\sim 300 \AA$ ), while 
the January data are closer to the two other more recent literature measurements (Gizis \& Bharat 2004; Mohanty, Jayawardhana, \& Barrado y Navascués 2003, EW of 28 and $42 \AA$ ), indicating that the changes which we observe for $2 \mathrm{M} 1207$ are not unique, and that variability persists on timescales of years.

2M1101: Four out of seven spectra for this brown dwarf show weak $\mathrm{H} \alpha$ emission with EW $\sim 15 \AA$, but in three consecutive exposures on March 18 and 19 the EW is increased by a factor of $\sim 6$ (see Fig. 2). This enhancement is also seen in $\mathrm{H} \beta$ and it coincides with the appearance of $\mathrm{H} \gamma$, HeI5875, and CaII lines, which are either very faint or not detected when $\mathrm{H} \alpha$ is weak. $\mathrm{H} \alpha$ and $\mathrm{H} \beta$ are clearly correlated. HeI6678 is only seen in the first spectrum, and neither of our spectra shows forbidden line or OI emission. We note that the only literature value for the $10 \%$ width measured by Muzerolle et al. $\left(2005,283 \mathrm{kms}^{-1}\right)$ is higher than any of our measurements, whereas their $\mathrm{EW}$ is comparable to our values.

RhoOph-32: The Balmer lines and both HeI lines are strong in all exposures, and their time evolution is comparable: All lines start on a high level, and their intensity is decreased in the last three datapoints. The best correlations are seen for $\mathrm{H} \alpha$ vs. HeI5875 and $\mathrm{H} \alpha$ vs. $\mathrm{H} \beta$. Additionally, we observe weak CaII triplett emission, which shows a trend comparable to $\mathrm{H} \alpha$. We do not detect emission in [SII], [OI], and OI. Natta et al. (2004) published an EW of $48 \AA$, which is at the low end of our datapoints. Their $10 \%$ width of $248 \mathrm{kms}^{-1}$, however, is comparable with our values.

ChaH $\alpha$ 1: The Balmer lines and the CaII triplet are clearly detected in all spectra. $\mathrm{H} \alpha$ and CaII behave very similarly, with a maximum in the third spectrum, and good correlations between the EWs. On the other hand, $\mathrm{H} \beta$ and $\mathrm{H} \gamma$ have their maximum in the fourth spectrum. Both HeI lines can only be seen in the last two exposures, and thus behave in a comparable manner to the higher Balmer lines. Clearly, there are two groups of lines, where the second group $(\mathrm{H} \beta, \mathrm{H} \gamma, \mathrm{HeI})$ appears to react delayed by a few hours with respect to the first group (H $\alpha$, CaII). In neither of our spectra do we find emission in [SII], [OI], OI. All literature EW and 10\% width measurements for $\mathrm{H} \alpha$ (Comerón, Neuhäuser, \& Kaas 2000; Luhman 2004; Natta et al. 2004; Mohanty, Jayawardhana, \& Basri 2005) are consistent with our results, indicating that $\mathrm{ChaH} \alpha 1$ does not show dramatic changes in the emission lines over timescales of a few years.

ISO217: The dominant lines are $\mathrm{H} \alpha$ and the CaII triplet, which are very strong in all exposures; the line ratio of the triplet is always close to 1:1:1. OI is clearly detected in all spectra, and $\mathrm{H} \beta$ and $\mathrm{H} \gamma$ in most of them. Both HeI lines are visible in the fourth spectrum, coinciding with the maximum in the higher Balmer lines, and in two out of five cases we also detect forbidden line emission in [SII]. The EW in $\mathrm{H} \alpha$, CaII, and [SII] behave comparably, with a maximum in the third spectrum. On the other hand, the higher Balmer lines, HeI, 
and OI are strongest in the fourth exposure, and are thus apparently delayed by $\sim 1 \mathrm{~h}$. $\mathrm{H} \alpha$ shows a clear correlation with $\mathrm{H} \beta$, and weak correlations with CaII and [SII]. Our $\mathrm{H} \alpha \mathrm{EW}$ and $10 \%$ measurements are consistent with literature values for this target (Luhman 2004; Muzerolle et al. 2005).

LS-RCrA 1: In our time series we see the Balmer lines, CaII triplet and [SII] in all spectra, and HeI5875 and OI in most of them. HeI6678, however, is not detected. The central CaII triplet line is clearly stronger than the other two, with an average line ratio of 1:2:1. CaII and [SII] EWs show the same trend: they increase in the first three spectra and drop off in the fourth exposure. In contrast, all other lines show an additional dip in the second spectrum. Correlations are seen for CaII vs. [SII], $\mathrm{H} \alpha$ vs. $\mathrm{H} \beta$ and $\mathrm{H} \alpha$ vs. HeI. Whereas the $\mathrm{H} \alpha 10 \%$ width is more or less constant and in agreement with literature values (Barrado y Navascués, Mohanty, \& Jayawardhana 2004), the EW varies strongly, although we do not observe the extreme values of $\sim 360 \AA$ and $\sim 240 \AA$ reported by Fernández \& Comerón (2001) and Barrado y Navascués, Mohanty, \& Jayawardhana (2004).

\section{Discussion of specific targets}

In the previous two sections we gave a purely phenomenological description of the emission line variability for our six targets. In this section, we will interpret these results, aiming to understand the origin of the variability and the line profiles. This has to be done for each target separately, due to differences in their behavior. We would like to emphasize that many of the conclusions in the following subsections have to be somewhat speculative, since the large number of free parameters usually allows more than one possible interpretation for the spectral variability.

\section{1. $2 \mathrm{M} 1207$}

2M1207, which is a likely member of the $~ 8$ Myr old TW Hydrae association has already been discussed in some detail in SJB05. Therefore, here we wish to recall the scenario used in this previous paper to explain the emission line variability. In SJB05 we discussed the pronounced red-shifted absorption component in the $\mathrm{H} \alpha$ profiles of $2005 \mathrm{March}$; this feature disappears and re-appears on a $\sim 1$-day timescale, comparable with the rotation period of the object. Since red-shifted absorption indicates that we see cool, absorbing gas projected against hot material, we interpreted this behaviour as a consequence of a nearly edge-on view, where the inclination between rotational axis and line of sight is $>60^{\circ}$. As the 
object rotates, the accretion funnel moves through the line of sight. When we see a strong red-shifted absorption feature, we may be looking into the accretion column. This view is supported by the evolution of the flux ratios between $\mathrm{H} \alpha$ and higher Balmer lines: In spectra with a strong red-shifted absorption component in $\mathrm{H} \alpha$, the line fluxes in the higher Balmer lines tend to be decreased relative to $\mathrm{H} \alpha$. Thus, if absorption is present, we see on average cooler gas than in the symmetric case, where the hot spot is unobscured.

Additionally, we found a significant increase in the $\mathrm{H} \alpha 10 \%$ width between the January and the March run, indicating an accretion rate change by a factor of 5-10. We would like to stress that this burst of accretion on timescales of six weeks is seen not only in $\mathrm{H} \alpha$; except for HeI6678, all other emission lines become significantly stronger in the March spectra. In fact, most of the variability in the EW is produced on timescales of weeks rather than days. Thus, the strong profile changes seen on timescales of hours in March do not significantly affect the global accretion properties of the object.

The variance profile (see Fig. 1) of 2M1207 clearly shows that most of the $\mathrm{H} \alpha$ variability is produced on the blue side of the profile with a strong peak at about $-100 \mathrm{kms}^{-1}$, although there are also peaks at zero and red-shifted velocities. Since strongly blue-shifted peaks in the variance profile indicate strong variability in the wind, this could simply mean that the increase seen in the $10 \%$ width is mainly due to a burst in a hot wind. On the other hand, although the total $10 \%$ width increases by about $70 \mathrm{kms}^{-1}$ between January and March, the difference between red and blue part of this value does not change significantly, as long as no second peak on the red side is visible (see Fig. 4). Therefore, the $10 \%$ width increases both on the red and on the blue side. The most likely interpretation is that we observe a change in the accretion rate accompanied by a hot wind.

In two spectra in March, some lines (He5875, $\mathrm{H} \beta, \mathrm{H} \gamma$ ) show a brief enhancement over a few hours, but $\mathrm{H} \alpha$ is not unusually strong. The origin of this event is unclear, it might be due to a flare in a hot chromospheric region, or another accretion-related burst.

\section{2. $2 \mathrm{M} 1101$}

2M1101, a candidate late-M type object in the ChaI star forming region, appears to be a non-accreting, weakly active brown dwarf in four out of seven spectra. In the remaining three spectra, however, we see a clear emission burst: EW and $10 \%$ width in $\mathrm{H} \alpha$ strongly increase, and reach levels sufficiently high to classify the object as an accretor. From the $10 \%$

width we estimate an accretion rate of about $10^{-11} M_{\odot} \mathrm{yr}^{-1}$ (Natta et al. 2004) during this burst. At the same time, other lines like CaII, H $\gamma$, HeI5875 appear in the spectra, which are 
at least partly related to the accretion process (see Sec. 2.2). Furthermore, the $\mathrm{H} \alpha$ profile exhibits a red absorption feature, which is clear evidence for infalling material. Thus, based on $10 \%$ width, $\mathrm{H} \alpha$ profile, and the appearance of additional emission indicators, we argue that this event is related to accretion and not, e.g., a flare of chromospheric activity.

The origin of this behaviour is unclear. It is unlikely that we just see rotationally modulated emission, i.e. a strongly asymmetric accretion flow, because the increase in the line widths happens within a few hours, whereas the high level is maintained for at least one day. A rotational modulation would imply a more periodic behaviour. To definitely exclude a rotational origin for the observed variability, a $v \sin i$ measurement (which is not possible based on our data, see Sect. 2.3) would be desirable.

The alternate explanation is a sudden increase of the accretion rate by at least one order of magnitude. We have seen a similar increase for 2M1207 (SJB05) on timescales of a few weeks; here we may have the first evidence for strong accretion rate changes on timescales of a few hours. Such behavior would be expected if the accretion is not continuous, but rather clumpy, with a typical timescale in the range of a few days for the discontinuity. Whether or not this applies to 2M1101 could be clarified by further time series observations with better sampling.

2M1101 has been observed before with high-resolution spectroscopy by Muzerolle et al. (2005). Remarkably, H $\alpha$ shows an extreme P Cygni profile with strong absorption on the blue side in their spectra from December 2003/January 2004. This has already been seen for some $\mathrm{T}$ Tauri stars with high mass-loss rates, and the usual interpretation is that the $\mathrm{H} \alpha$ emission is predominantly formed in the wind. In contrast with this literature spectrum, our spectra show only weak evidence for outflowing material as an asymmetry in the $\mathrm{H} \alpha$ line profile (see Sect. 3). This implies that both the infall and outflow rates are highly variable with time, again underlying the need for more detailed follow-up observations.

\section{3. $\rho \mathrm{Oph}-32$}

$\rho$ Oph-32 has broad emission lines, yet no dramatic line profile and intensity variations are seen within our time series. The $\mathrm{H} \alpha$ width exceeds $200 \mathrm{kms}^{-1}$ in all spectra, implying accretion rates between $10^{-10}$ and $10^{-11} M_{\odot \mathrm{yr}^{-1}}$ (Natta et al. 2004), and the asymmetry in the profile as well as the persistent emission in CaII support that $\rho$ Oph-32 is an active accretor, in agreement with previous studies of this object.

In contrast to the literature spectrum of Natta et al. (2004), we see no forbidden line emission and no redshifted absorption in $\mathrm{H} \alpha$. Instead, our $\mathrm{H} \alpha$ profiles show a weak 'shoulder' 
on the blue side, which may be due to a weak blue-shifted absorption feature. Thus, there is only weak if any evidence for wind in our spectra. We conclude that outflow and accretion in $\rho$ Oph-32 are probably variable on timescales of years, but more or less constant over a few weeks.

An appealing interpretation for the lack of variability is a face-on geometry for the disk and the central object. In this case, rotation can be excluded as a major cause of variability, since we see always the same hemisphere of the object. $\rho$ Oph-32 exhibits strong near/mid-infrared colour excess indicative of a disk (Persi et al. 2000; Natta et al. 2002), which also favours a pole-on view because the surface area of the disk visible to the observer is maximized when viewed face-on. An alternate explanation for the lack of variability would be a more or less symmetric field configuration implying axially symmetric accretion.

\subsection{ChaH $\alpha-1$}

The literature data for $\mathrm{ChaH} \alpha-1$ are ambiguous with respect to the accretion status of this object. Comerón, Neuhäuser, \& Kaas (2000) and Luhman (2004) classified it as weakly accreting based on $\mathrm{H} \alpha \mathrm{EW}$, but the low $10 \%$ widths measured by Natta et al. (2004) and Mohanty, Jayawardhana, \& Basri (2005) led them to suggest that the H $\alpha$ emission of $\mathrm{ChaH} \alpha-1$ is predominantly caused by chromospheric activity. Our $\mathrm{H} \alpha$ profiles of $\mathrm{ChaH} \alpha-1$ indeed resemble profiles of chromospherically active stars; it is more or less symmetric, not extraordinarily broad and exhibits a small central absorption dip, which is usually interpreted as self-absorption in the chromosphere. The $10 \%$ widths are clearly below the threshold for ongoing accretion, indicating that accretion probably does not contribute significantly to the emission line flux. However, we detect HeI6678 in emission in one of our spectra with an EW of $1.6 \AA$, clearly too high to be explained only by activity (see Sect. 2.2). Moreover, the EW in $\mathrm{H} \alpha$ is very high compared with non-accreting, active objects with similar spectral type (Barrado y Navascués \& Martin 2003). Thus, we cannot rule out that ChaH $\alpha-1$ is weakly accreting, in addition to being chromospherically active. Near- and mid-infrared color excesses provide additional support for a disk surrounding this object (Persi et al. 2000; Kenyon \& Gómez 2001; Jayawardhana et al. 2003).

There are at least two possible ways to explain why we observe two different groups of emission lines (see Sec. 4). Let us first assume that all emission is produced by chromospheric activity. In this scenario, the object may harbor two large active regions in the chromosphere with different temperatures. One of these regions is mainly responsible for the 'high temperature lines' like HeI and the higher Balmer features. The second region is cooler and thus does not contribute to the high-energy features. $\mathrm{H} \alpha$ and CaII are generated in both 
regions. The 'delay' seen in the time evolution of both groups of lines can be explained with a phase-difference between the two regions. A similar scenario is possible for the case where $\mathrm{ChaH} \alpha-1$ is weakly accreting. Here, the object would have a hot accretion flow, which may be responsible for the emission in $\mathrm{HeI}, \mathrm{H} \beta$, and $\mathrm{H} \gamma$, and an active chromospheric spot. If this active region appears a few hours earlier on the visible hemisphere than the accretion hot spot, we would expect a delay in the high-energy lines.

In both scenarios, the variability in the emission lines would be due to rotation. More evidence for this interpretation comes from Fig. 4, where we see a hint of a period in the evolution of the asymmetry in $\mathrm{H} \alpha$. This is expected, because both accretion and activity features should change from blue- to redshifted as they move through the line of sight. From the rotational velocity given by Joergens \& Guenther (2001), we derive an upper limit for the rotation period of $3.1 \mathrm{~d}$ (using radii from Baraffe et al. (1998)). The spectral variability implies a period in the range of a few days, consistent with the observed rotational velocity.

We tentatively conclude that the modest variability of $\mathrm{ChaH} \alpha-1$ is due to rotation. This would additionally explain the large discrepancy in the literature values for the $\mathrm{H} \alpha \mathrm{EW}$, which were derived from single spectra, because the emission line intensity would depend strongly on the rotational phase. The interpretation of rotationally modulated emission lines implies an asymmetry in the chromospheric active regions and (perhaps also) in the accretion geometry, as well as a non-negligible inclination between the rotational axis and the line of sight.

\subsection{ISO217}

This ChaI object at the substellar boundary is known to have intense and strongly asymmetric $\mathrm{H} \alpha$ emission (Luhman 2004; Muzerolle et al. 2005). Our spectra confirm this finding; they show broad and asymmetric $\mathrm{H}$ Balmer features, clearly indicative of ongoing accretion. Forbidden line emission provides evidence for outflowing material as well, as also implied by the presence of an $\mathrm{H} \alpha$ absorption feature that appears clearly blue-shifted in most spectra. Correlations between the EW in [SII], $\mathrm{H} \alpha$, and CaII show that winds influence $\mathrm{H} \alpha$ and CaII somewhat, whereas the 'high-energy' lines which do not show any connection to [SII] are probably dominated by accretion.

ISO217 clearly shows variability in both accretion and wind related features. In comparison to 2M1207 and 2M1101, however, the changes in EW and 10\% width are moderate, i.e. the changes in the accretion rate are well below an order of magnitude. Given our sparse sampling, this does not, of course, exclude the possibility of more dramatic changes in its 
accretion behavior. The variability of ISO217 could be due to rotation, since it occurs on timescales of a few hours to days, comparable to typical rotation periods for such objects.

As can be seen in Fig. 1, the variability in $\mathrm{H} \alpha$ appears to be dominated by the emission wings and thus the high-velocity infalling gas. Additionally, there is a variation of the relative strenghts of the two peaks, which is probably a consequence of a velocity change in the windgenerated absorption component. As we have shown in our decomposition procedure, the absorption moves towards the blue side within our time series by about $20 \mathrm{kms}^{-1}$ (Sec. 3), which gradually suppresses the flux on the blue side of the profile, leading to an asymmetry of the peaks.

Perhaps the most interesting feature in our time series is the short time delay of $\sim 1 \mathrm{~h}$ between HeI and higher Balmer lines and $\mathrm{H} \alpha$, CaII, [SII]. As argued above, the difference between the two groups of lines is that the second group either exclusively or partly formed in outflows. Thus, the time delay may be explained by a time-lag between wind and accretion. One explanation for such behaviour is a coupling between outflow and infall rates, as already seen in T Tauri stars (e.g. for DF Tau, Johns-Krull \& Basri 1997). As in DF Tau, the windrelated feature in ISO217 increases before the accretion-related lines. This is expected in a scenario where the wind originates from somewhat cooler regions at the inner edge of the disk, whereas the high-energy accretion features are formed very close to the surface of the object. In this case, the time-lag would correspond to the time needed for the infalling material to travel from the inner disk-region, where the wind forms, to the object. Assuming free-fall velocity, the distance between the inner disk boundary (in the magnetospheric accretion scenario, the distance at which the disk is truncated by the magnetosphere) and the surface of the object would then be in the range of $5 \cdot 10^{5} \mathrm{~km}$ or about one object radius, a value which is not implausible for substellar objects (Muzerolle et al. 2003).

A time-lag between an observed increase in wind and accretion can also be explained by rotation, if wind and accretion regions are both asymmetric and spatially offset. Since the wind is believed to be collimated by the magnetic field, the effect could be enhanced if the axis of the magnetic field is inclined with respect to the rotational axes, introducing an additional asymmetry to the system.

\subsection{LS-RCrA 1}

The striking emission line spectrum of this object at the substellar boundary has been reported by Fernández \& Comerón (2001) and Barrado y Navascués, Mohanty, \& Jayawardhana (2004). It shows all spectroscopic signs of ongoing accretion as well as evidence for mass 
outflow. This is confirmed by our data: The $\mathrm{H} \alpha 10 \%$ width is well above the limit between accretors and non-accretors in all spectra, and other indicators of accretion and outflow are also seen. Based on the $10 \%$ width, we estimate an accretion rate of about $10^{-10} M_{\odot} \mathrm{yr}^{-1}$, which is at the lower end of the range given in the literature (Barrado y Navascués, Mohanty, \& Jayawardhana 2004).

The CaII triplet shows a clear deviation from the 1:1:1 line ratio expected for an accretion-dominated origin; furthermore, it is correlated with [SII]. Thus, the CaII emission is probably at least partly formed in the wind, and not only in the accretion flow. $\mathrm{H} \alpha$, on the other hand, behaves in the same manner as other accretion-related features, and it shows no evidence for blue-shifted absorption, indicating that the line originates primarily in the accretion flow.

LS-RCrA 1 is one of the less variable targets in our sample; the $\mathrm{H} \alpha$ line is more or less symmetric, and does not show significant variability in the profile shape or the $10 \%$ width. This may indicate that the system is either seen close to face-on or that the accretion flow is fairly symmetric; both scenarios would avoid rotational modulation. Based on our time series, we favour the latter explanation, because we see strong forbidden line emission, but no significant wind signature in $\mathrm{H} \alpha$. which may imply that the $\mathrm{H} \alpha$ and forbidden line emission regions are spatially offset. With a face-on geometry, it is more likely that the [SII] and $\mathrm{H} \alpha$ emitting regions overlap in the line of sight, producing a wind signature in $\mathrm{H} \alpha$. On the other hand, with the disk seen close to edge-on, it is much easier to imagine that the outflow is seen against the background sky and not against the accretion flow and the object itself, and is thus spatially offset from the $\mathrm{H} \alpha$ emitting regions, which would explain the lack of blue-shifted absorption in $\mathrm{H} \alpha$.

The published studies are inconclusive about the disk orientation of LS-RCrA 1. On the one hand, there is some evidence in the literature for an edge-on geometry for LS-RCrA 1, because a) the object shows no colour excess in the near-infrared although it is accreting, b) it appears to be underluminous, as would be expected if the object is obscured by the disk, and c) the outflow signature is very prominent (see Fernández \& Comerón 2001; Barrado y Navascués, Mohanty, \& Jayawardhana 2004). However, the forbidden line emission is asymmetric and blue-shifted, indicating that the receding jet may be obscured by the disk, which would argue against an edge-on scenario (Fernández \& Comerón 2005). Thus, based on currently available data, it is not possible to distinguish reliably between face-on and edge-on geometries. 


\section{General implications}

Although our six targets are rather diverse in terms of their accretion properties, some general conclusions are possible. We presented in this paper the first dedicated spectroscopic variability study for accreting brown dwarfs, albeit with sparse sampling for most objects. Perhaps the most important result from this program is that accreting brown dwarfs, similar to $\mathrm{T}$ Tauri stars, are strongly variable, both in broad band photometric time series (Scholz \& Eislöffel 2004, 2005) and in their emission line spectrum. Variability was originally the main observational feature of $\mathrm{T}$ Tauri stars, leading to a systematic study of these objects. It is therefore worth confirming that variability persists down to sub-stellar masses. This

completes the recently emerged picture of young brown dwarfs as very low mass T Tauri-like objects, which share many properties with accreting stars.

The broad emission lines of accreting BDs show a large variety of shapes; we observe profiles ranging from double-peaked to more or less symmetric, as is the case for more massive stars. The intensity and profile shapes are variable on timescales ranging from hours to years, again in total agreement with CTTS. One implication from this finding is a cautionary note: since accretion-related properties can be highly variable, investigations based on one single spectrum or datapoint are not particularly reliable. To study accretion properties, we either have to work with large samples of objects (to average over the variability) or with time series.

One particularly important recent finding, which may be strongly influenced by variability, is the relationship between object mass and accretion rate. White \& Basri (2003) pointed out a positive correlation between $M$ and $\dot{M}$, which has been specified more recently as $\dot{M} \propto M^{2}$ (Natta et al. 2004; Mohanty, Jayawardhana, \& Basri 2005; Muzerolle et al. 2005). This relationship has been established using a fairly large number of objects, and in all samples the accretion rates in a given mass bin scatter by about one order of magnitude. In fact, it is only possible to see the accretion rate vs. mass correlation when sub-stellar objects are included, and thus the object masses cover more than one order of magnitude. (Otherwise the scatter is too large.) The derived relationship has been interpreted recently as a direct consequence of Bondi-Hoyle accretion, implying that the accretion process in the inner disk is governed by the large-scale environment of the parent cloud (Padoan et al. 2005). Although this approach is able to reproduce the observed correlation, the scatter around the correlation remains unexplained.

Variability provides a straightforward solution for this problem. Two out of six targets (2M1207 and 2M1101) in our sample show accretion rate variations by about one order of magnitude. Since we intentionally selected objects where we expected to see strong changes, these may be extreme cases of accretion rate variability. On the other hand, given our 
sparse sampling, our variability estimates should be considered only as lower limits; we cannot exclude that our targets show larger accretion rate changes on longer timescales. Thus, we conclude that brown dwarfs can in some cases show accretion rate variations of at least one order of magnitude.

In the Bondi-Hoyle scenario, all objects with a given mass should have the same (average) accretion rate. Due to variability, the accretion rates based on single spectra will scatter by about one order of magnitude around this average value. Additional noise may be introduced by geometry and obscuration effects. Thus, the scatter observed in the massaccretion rate relationship is not surprising - and it demonstrates that variability studies are an important complement to understand the accretion behaviour of BDs.

Variability information can be used to constrain the nature of accretion and winds/outflows in sub-stellar objects, in particular to probe the magnetospheric accretion scenario, which allows for asymmetric accretion and winds, in contrast to spherical infall. In general, there are two ways to produce variability in the emission lines: either the accretion and/or the wind is not steady, which may occur in a scenario of stochastic, clumpy flows, and does not necessarily require magnetic funneling, or the magnetic field, which funnels the flow, is highly asymmetric, leading to rotationally modulated accretion and wind. It is possible to distinguish between the two scenarios by from the shape of emission lines - if the features look asymmetric and the profiles change with time, we would expect an asymmetric field geometry. That, in turn, would suggest non-spherical accretion, and thus provide indirect evidence for magnetospheric accretion. We note that this argument is not reversible: the absence of emission line variability or profile asymmetry does not rule out asymmetric accretion.

Among our targets, at least two show strong emission line variability, both in intensity and profile shape, and an asymmetric $\mathrm{H} \alpha$ feature (2M1207 and ISO217). A third one (2M1101) exhibits an asymmetric profile in parts of the time series and a strong accretion burst, but as argued in Sec. 5 the timescales of this event are barely compatible with rotational modulation. Thus, at least for one third of our objects, an asymmetric flow geometry is required to explain the emission line behaviour, providing a strong case for magnetic funneling of accretion and winds.

Based on the available data, we can obtain an estimate of the magnetic field strengths necessary for magnetospheric accretion in sub-stellar objects, using the relationship given by Hartmann (2002) (Equ. 1), and scaling it from the stellar regime to the properties of BDs. As as cautious note, we would like to add that this should only be considered as an order of magnitude estimate, mainly because of the limitations of the equation in Hartmann (2002). For example, this relationship assumes a dipolar field geometry, whereas there is 
clear observational evidence for more complex field structure in T Tauri stars (e.g. JohnsKrull \& Gafford 2002). Given the absence of magnetic field measurements for brown dwarfs, a first estimate of the field strength - albeit crude - is still useful.

We approximate the mass of a typical BD to be about $1 / 20$ of a solar mass and the radius to be about $1 / 3$ (at $1 \mathrm{Myr}$ ). Addtionally, we scale the accretion rate following $\dot{M} \propto M^{2}$, and assume that the radius at which the disk is truncated by the magnetic field is (in units of the object radius) more or less constant with mass. The latter assumption is in line with the results from profile modeling by Muzerolle et al. (2003), where reasonable agreement between observed and modeled profiles can be achieved without major mass dependency in the (relative) size of the magnetosphere. With these prerequisites, we estimate that the ratio of the surface magnetic field strength on accreting BDs and stars is $\sim 0.6$. Since T Tauri stars have in many cases magnetic fields of several kG (e.g. Symington et al. 2005), this result indicates that young substellar objects may host magnetic fields in the range of $1 \mathrm{kG}$. Although the uncertainties in the given estimate are considerable (see above), we tentatively conclude that magnetic field strengths for BDs are probably in the same order of magnitude as for stars.

\section{Conclusions}

This paper contains the first spectroscopic variability study for young accreting brown dwarfs. We obtained, depending on the target, 4-13 high-resolution spectra using the Magellan/Clay $6.5 \mathrm{~m}$ telescope, covering timescales from a few hours to several weeks. Our targets are six sub-stellar objects in nearby star-forming regions, for which signs of accretion had been noted in previous studies. We measured line widths for the most prominent emission lines, either related to accretion, winds, or both. In all spectra, the continuum is faint, but not seriously affected by incomplete background subtraction or accretion veiling.

All our targets are variable in the emission lines. The most dramatic changes are seen for 2M1207, 2M1101, and ISO217, whereas the remaining targets ( $\rho$ Oph-32, ChaH $\alpha-1$, LSRCrA 1) show significantly less variability. The most prominent line in all cases is the $\mathrm{H} \alpha$ feature. Our targets exhibit a variety of $\mathrm{H} \alpha$ profiles, similarly to accreting $\mathrm{T}$ Tauri stars. Two objects (2M1207, ISO217) have strongly asymmetric and double-peaked profiles at least in parts of the time series, produced by a superposition of broad emission and either a red- (2M1207) or blue-shifted absorption feature. 2M1101 also exhibits a weak red-shifted absorption feature in parts of our time series.

Strong changes in the $\mathrm{H} \alpha 10 \%$ width are seen in 2M1207 and 2M1101, indicating vari- 
ations in the accretion rate on timescales of weeks (2M1207) and hours (2M1101). In both cases, this finding is supported by additional accretion indicators in the spectrum. For $2 \mathrm{M} 1101$, the variations are consistent with a scenario of non-steady, clumpy accretion. We also see evidence for strong variability in the outflow rate in at least three targets (2M1101, $\rho$ Oph-32, ISO217), particularly if we compare our results with literature data. In two cases (2M1207, ISO217) there are indications for a coupling between infall and outflow rate changes. In some cases the variability is best explained by rotational modulation of the emission line flux. For LS-RCrA 1, the variability characterictic supports a close to edge-on view of the disk.

One important result from this study is a cautionary note: given that most accreting targets are strongly variable, studies of accretion-related properties have to be based either on large samples or on time series. One example is the recently found accretion rate vs. mass relationship, which shows a suspiciously large scatter. We demonstrate that it is possible to explain this noise by taking into account variability information.

For at least two out of six targets, we have to assume asymmetric accretion flows to interpret the emission line shape and variations. This indirectly supports funneling of the accretion, as predicted in the magnetospheric accretion scenario for T Tauri stars, and thus provides evidence that this scenario also applies to brown dwarfs. This implies the existence of large-scale magnetic fields, which may have $\sim \mathrm{kG}$ field strengths, in accreting substellar objects.

Some of the observations were carried out by Alexis Brandeker, whose help is gratefully acknowledged. We thank the Las Campanas Observatory staff for their assistance. The constructive comments from an anonymous referee helped to improve the paper. This research was supported by an NSERC grant and University of Toronto startup funds to R.J.

\section{REFERENCES}

Alencar, S. H. P., Batalha, C. 2002, ApJ, 571, 378

Allard, F., Hauschildt, P. H., Schwenke, D. 2000, ApJ, 540, 1005

Barrado y Navascués, D., Jayawardhana, R. 2004, ApJ, 615, 840

Barrado y Navascués, D., Mohanty, S., Jayawardhana, R. 2004, ApJ, 604, 284

Barrado y Navascués, D., Martin, E. L. 2003, AJ, 126, 2997 
Baraffe, I., Chabrier, G., Allard, F., Hauschildt, P. H. 2002, A\&A, 382, 563

Baraffe, I., Chabrier, G., Allard, F., Hauschildt, P. H. 1998, A\&A, 337, 403

Basri, G., Batalha, C. 1990, ApJ, 363, 654

Bate, M. R.; Bonnell, I. A., Bromm, V. 2002, MNRAS, 332, 65

Beristain, G., Edwards, S., Kwan, J. 2001, ApJ, 551, 1037

Bontemps, S., André, P., Kaas, A. A., Nordh, L., Olofsson, G., et al. 2001, A\&A, 372, 173

Bouvier, J., Grankin, K. N., Alencar, S. H. P., Dougados, C., Fernández, M., et al. 2003, A\&A, 409, 169

Bouvier, J., Chelli, A., Allain, S., Carrasco, L., Costero, R., et al. 1999, A\&A, 349, 619

Comerón, F., Neuhäuser, R., Kaas, A. A., 2000, A\&A, 359, 269

Doppmann, G. W., Greene, T. P., Covey, K. R., Lada, C. L. 2005, AJ, 130, 1145

Fernández, M., Comerón, F. 2005, A\&A, 440, 1119

Fernández, M., Comerón, F. 2001, A\&A, 380, 264

Fernández, M., Ortiz, E., Eiroa, C., Miranda, L. F. 1995, A\&AS, 114, 439

Gizis, J. E., Bharat, R. 2004, ApJ, 608, 113

Gizis, J.E. 2002, ApJ, 575, 484

Gizis, J. E., Reid, I. N., Hawley, S. L. 2002, AJ, 123, 3356

Graham, J. A., Heyer, M. H. 1988, PASP, 100, 1529

Hartmann, L. 2002, ApJ, 566, 29

Hartmann, L., Hewett, R., Calvet, N. 1994, ApJ, 426, 669

Herbst, W., Herbst, D. K., Grossman, E. J., Weinstein, D., 1994, AJ, 108, 1906

Hirth, G. A., Mundt, R., Solf, J. 1997, A\&AS, 126, 437

Jayawardhana, R., Ardila, D. R., Stelzer, B., Haisch, K. E., Jr. 2003, AJ, 126, 1515

Jayawardhana, R., Mohanty, S., Basri, G. 2003, ApJ, 592, 282 
Jayawardhana, R., Mohanty, S., Basri, G. 2002, ApJ, 578, 141

Joergens, V. 2005, A\&A, in press

Joergens, V., Fernández, M., Carpenter, J. M., Neuhäuser, R. 2003, ApJ, 594, 971

Joergens, V., Guenther, E. 2001, A\&A, 379, 9

Johns-Krull, Ch. M., Gafford, A. D. 2002, ApJ, 573, 685

Johns-Krull, Ch., Basri, G., 1997, ApJ, 474, 433

Johns, C., Basri, G. 1995, AJ, 109, 2800

Kenyon, S. J., Gómez, M. 2001, AJ, 121, 2673

Koenigl, A. 1991, ApJ, 370, 39

Kroupa, P., Bouvier, J. 2003, MNRAS, 346, 369

Luhman, K. L., Stauffer, J. R., Muench, A. A., Rieke, G. H., Lada, E. A., Bouvier, J., Lada, C. J. 2003, ApJ, 593, 1093

Luhman, K. L. 2004, ApJ, 602, 816

Matt, S., Pudritz, R. E. 2005, MNRAS, 356, 167

Mitskevich, A. S., Natta, A., Grinin, V. P., 1993, ApJ, 404, 751

Mohanty, S., Jayawardhana, R., Basri, G. 2005, ApJ, 626, 498

Mohanty, S., Jayawardhana, R., Natta, A., Fujiyoshi, T., Tamura, M., Barrado y Navascués, D. 2004, ApJ, 609, 33

Mohanty, S., Jayawardhana, R., Barrado y Navascués, D. 2003 ApJ, 593, 109

Muzerolle, J., Luhman, K. L., Briceno, C., Hartmann, L., Calvet, N. 2005, ApJ, 625, 906

Muzerolle, J., Hillenbrand, L., Calvet, N., Briceno, C., Hartmann, L. 2003, ApJ, 592, 266

Natta, A., Testi, L., Muzerolle, J., Randich, S., Comerón, F., Persi, P. 2004, A\&A, 424, 603

Natta, A., Testi, L., Comerón, F., Oliva, E., D’Antona, F., Baffa, C., Comoretto, G., Gennari, S. 2002, A\&A, 393, 597

Padoan, P., Kritsuk, A., Norman, M. L., Nordlund, A. 2005, ApJ, 622, 61 
Padoan, P., Nordlund, A. 2004, ApJ, 617, 559

Pascucci, I., Apai, D., Henning, Th., Dullemond, C. P. 2003 ApJ, 590, 111

Persi, P., Marenzi, A. R., Olofsson, G., Kaas, A. A., Nordh, L., et al. 2000, A\&A, 357, 219

Reipurth, B., Pedrosa, A., Lago, M. T. V. T., 1996, A\&AS, 120, 229

Reipurth, B., Bally, J., Graham, J. A., Lane, A. P., Zealey, W. J. 1986, A\&A, 164, 51

Romanova, M. M., Ustyugova, G. V., Koldoba, A. V., Wick, J. V., Lovelace, R. V. E. 2003, ApJ, 595, 1009

Saar, S. H., Huovelin, J., Osten, R. A., Shcherbakov, A. G. 1997, A\&A, 326, 741

Scholz, A., Jayawardhana, R., Brandeker, A. 2005, ApJ, 629, 41 (SJB05)

Scholz, A., Eislöffel, J. 2005, A\&A, 429, 1007

Scholz, A., Eislöffel, J. 2004, A\&A, 419, 249

Shu, F., Najita, J., Ostriker, E., Wilkin, F., Ruden, S., Lizano, S. 1994, ApJ, 429, 781

Simon, M., Dutrey, A., Guilloteau, S. 2000, ApJ, 545, 1034

Symington, N. H., Harries, T. J., Kurosawa, R., Naylor, T. 2005, MNRAS, 358, 977

Uzdensky, D. A. 2002, ApJ, 572, 432

Whelan, E. T., Ray, T. P., Bacciotti, F., Natta, A., Testi, L., Randich, S., 2005, Nature, 435,652

White, R. J., Basri, G. 2003, ApJ, 582, 1109

Whitworth, A. P., Goodwin, S. P. 2005, Mem. Soc. Astron. Italiana, 76, 211 
Table 1. Target properties

\begin{tabular}{llccccc}
\hline \hline \multicolumn{1}{c}{ Full name } & Short name & $\mathrm{SpT}^{\mathrm{a}}$ & $v_{\text {rad }}\left(\mathrm{kms}^{-1}\right)^{\mathrm{a}}$ & $v_{\text {rot }}\left(\mathrm{kms}^{-1}\right)^{\mathrm{a}}$ & No. $^{\mathrm{b}}$ & ${\mathrm{H} \alpha \mathrm{VI}^{\mathrm{c}}}$ \\
\hline 2MASS J1207334-393254 & $2 \mathrm{M} 1207$ & $\mathrm{M} 8$ & 11.2 & 13 & 13 & 29 \\
2MASS J11013205-7718249 & 2M1101 & $\mathrm{M} 8$ & $\sim 15^{\mathrm{d}}$ & - & 7 & 49 \\
$\rho$ Oph-ISO032 & $\rho$ Oph-32 & $\mathrm{M} 7.5$ & $\sim 0^{\mathrm{e}}$ & - & 5 & 17 \\
ChaH $\alpha$-1 & $\mathrm{ChaH} \alpha-1$ & $\mathrm{M} 7.5$ & 15.5 & 7.6 & 5 & 19 \\
ChaI-ISO217 & ISO217 & M6.25 & $\sim 15^{\mathrm{d}}$ & - & 5 & 39 \\
LS-RCrA 1 & LS-RCrA 1 & $\mathrm{M} 6.5$ & $\sim 2$ & 18 & 4 & 16 \\
\hline
\end{tabular}

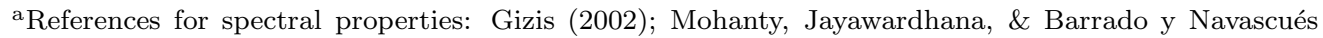
(2003); Muzerolle et al. (2005); Natta et al. (2002); Comerón, Neuhäuser, \& Kaas (2000); Joergens \& Guenther (2001); Joergens (2005); Mohanty, Jayawardhana, \& Basri (2005); Luhman (2004); Fernández \& Comerón (2001); Barrado y Navascués, Mohanty, \& Jayawardhana (2004)

${ }^{\mathrm{b}}$ Number of spectra in the time series

${ }^{\mathrm{c}} \mathrm{H} \alpha$ variability index, as defined in Sect. 3

d Average radial velocity of ChaI brown dwarfs

${ }^{\mathrm{e}} v_{\text {rad }}$ derived in this paper 
Table 2. Observing log and $\mathrm{H} \alpha$ measurements

\begin{tabular}{|c|c|c|c|c|c|c|c|c|}
\hline Date 2005 & UT & Slit & Binning & Target & Exp.time (s) & $\mathrm{H} \alpha \operatorname{EW}(\AA)^{\mathrm{a}}$ & $10 \%$ width $\left(\mathrm{kms}^{-1}\right)^{\mathrm{b}}$ & Comment \\
\hline $29 / 01$ & $05: 25$ & $0^{\prime \prime} 35$ & $1 \times 1$ & 2M1207 & $1 \times 900$ & 110 & 215 & \\
\hline $29 / 01$ & 08:08 & & & 2M1207 & $1 \times 1200$ & 64 & 215 & \\
\hline $01 / 02$ & $05: 56$ & & & 2M1207 & $1 \times 1200$ & 86 & 209 & \\
\hline \multirow[t]{6}{*}{$17 / 03$} & $00: 38$ & $1^{\prime \prime} 0$ & $2 \times 2$ & $2 \mathrm{M} 1207$ & $3 \times 600$ & 334 & 308 & double-peaked \\
\hline & $01: 15$ & & & $2 \mathrm{M} 1101$ & $3 \times 600$ & 22 & 131 & \\
\hline & $06: 45$ & 0.7 & & $\rho$ Oph-32 & $3 \times 1200$ & 88 & 270 & \\
\hline & $08: 14$ & $1{ }^{\prime \prime} 0$ & & $2 \mathrm{M} 1207$ & $3 \times 600$ & 387 & 274 & \\
\hline & $08: 52$ & & & $2 \mathrm{M} 1101$ & $3 \times 600$ & 17 & 135 & \\
\hline & $09: 28$ & & & LS-RCrA 1 & $2 \times 600$ & 80 & 270 & \\
\hline \multirow[t]{10}{*}{$18 / 03$} & $00: 23$ & $1 . \prime 0$ & $2 \times 2$ & $2 \mathrm{M} 1207$ & $3 \times 600$ & 259 & 291 & double-peaked \\
\hline & $01: 14$ & & & 2M1101 & $3 \times 900$ & 12 & 122 & \\
\hline & 02:06 & & & ISO217 & $3 \times 600$ & 109 & 359 & \\
\hline & $02: 41$ & & & $\mathrm{ChaH} \alpha-1$ & $3 \times 600$ & 72 & 139 & \\
\hline & $05: 16$ & & & $\mathrm{ChaH} \alpha-1$ & $3 \times 600$ & 74 & 135 & \\
\hline & $05: 50$ & & & ISO217 & $3 \times 900$ & 142 & 376 & \\
\hline & $06: 46$ & & & $\rho \mathrm{Oph}-32$ & $3 \times 1200$ & 82 & 224 & \\
\hline & 08:03 & & & $2 \mathrm{M} 1207$ & $3 \times 600$ & 229 & 253 & \\
\hline & $08: 42$ & & & $2 \mathrm{M} 1101$ & $3 \times 600$ & 92 & 232 & accretion burst \\
\hline & 09:19 & & & LS-RCrA 1 & $3 \times 600$ & 44 & 266 & \\
\hline \multirow[t]{12}{*}{$19 / 03$} & $23: 52$ & $1^{\prime \prime} 0$ & $2 \times 2$ & $\mathrm{ChaH} \alpha-1$ & $3 \times 600$ & 180 & 165 & \\
\hline & $00: 28$ & & & $2 \mathrm{M} 1207$ & $3 \times 600$ & 279 & 283 & double-peaked \\
\hline & 02:06 & & & $2 \mathrm{M} 1101$ & $3 \times 900$ & 85 & 224 & accretion burst \\
\hline & 03:07 & & & ISO217 & $3 \times 600$ & 226 & 367 & \\
\hline & $03: 57$ & & & $\mathrm{ChaH} \alpha-1$ & $3 \times 600$ & 142 & 143 & \\
\hline & $04: 32$ & & & $2 \mathrm{M} 1207$ & $3 \times 600$ & 308 & 257 & double-peaked \\
\hline & 05:07 & & & ISO217 & $3 \times 600$ & 219 & 363 & \\
\hline & 06:06 & & & $\mathrm{ChaH} \alpha-1$ & $2 \times 600$ & 132 & 152 & \\
\hline & $06: 37$ & & & $\rho$ Oph-32 & $3 \times 1200$ & 100 & 224 & \\
\hline & $07: 50$ & & & $2 \mathrm{M} 1207$ & $3 \times 600$ & 258 & 270 & \\
\hline & $08: 27$ & & & 2M1101 & $3 \times 900$ & 126 & 194 & accretion burst \\
\hline & $09: 29$ & & & LS-RCrA 1 & $3 \times 600$ & 125 & 291 & \\
\hline \multirow[t]{2}{*}{$27 / 03$} & $03: 56$ & $0 .{ }^{\prime \prime} 35$ & $1 \times 1$ & 2M1207 & $1 \times 1800$ & 192 & 295 & double-peaked \\
\hline & $06: 29$ & & $2 \times 2$ & $\rho$ Oph-32 & $1 \times 1200$ & 85 & 241 & \\
\hline $28 / 03$ & $04: 46$ & $0^{\prime \prime} 35$ & $1 \times 1$ & 2M1207 & $1 \times 1800$ & 183 & 279 & double-peaked \\
\hline \multirow[t]{4}{*}{$29 / 03$} & $04: 38$ & $00^{\prime \prime} 35$ & $1 \times 1$ & $2 \mathrm{M} 1207$ & $1 \times 1800$ & 261 & 285 & double-peaked \\
\hline & $05: 14$ & & $2 \times 2$ & ISO217 & $1 \times 1800$ & 73 & 342 & \\
\hline & $05: 47$ & & & 2M1101 & $1 \times 1800$ & 13 & 127 & \\
\hline & $07: 54$ & & & $\rho$ Oph-32 & $1 \times 1800$ & 50 & 211 & \\
\hline \multirow[t]{2}{*}{$30 / 03$} & $05: 12$ & $0^{\prime \prime} 35$ & $1 \times 1$ & $2 \mathrm{M} 1207$ & $1 \times 1500$ & 322 & 304 & double-peaked \\
\hline & $08: 11$ & & $2 \times 2$ & LS-RCrA 1 & $1 \times 1800$ & 71 & 304 & \\
\hline
\end{tabular}

aThe average measurement error in EW is $\sim 1 \AA$.

${ }^{\mathrm{b}}$ The measurement error in $10 \%$ width is $\sim 5 \mathrm{kms}^{-1}$ 

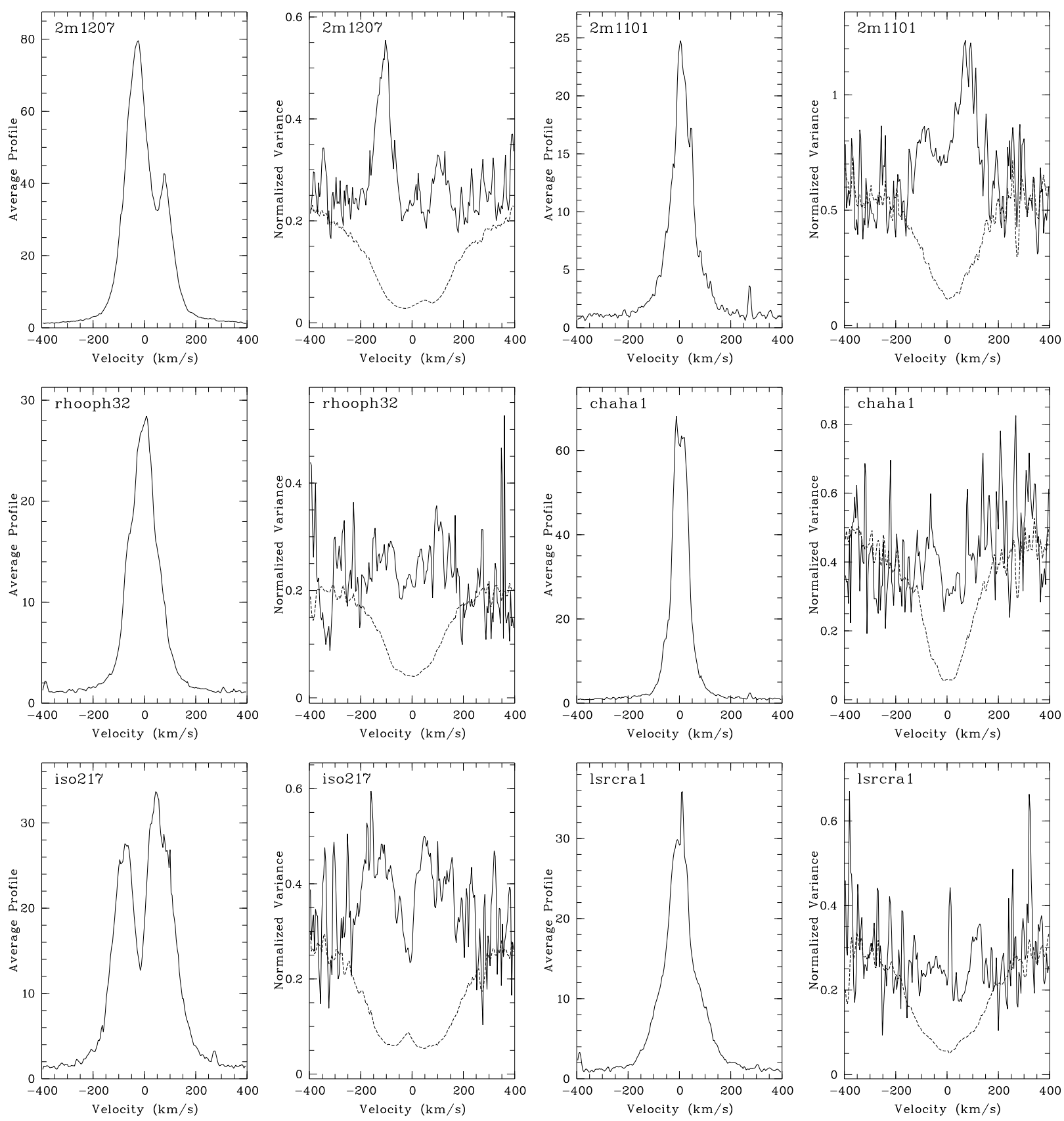

Fig. 1.- Average and normalised variance $\mathrm{H} \alpha$ profiles. Dashed lines show the level of zero variability (see Sect. 3 for explanation). 

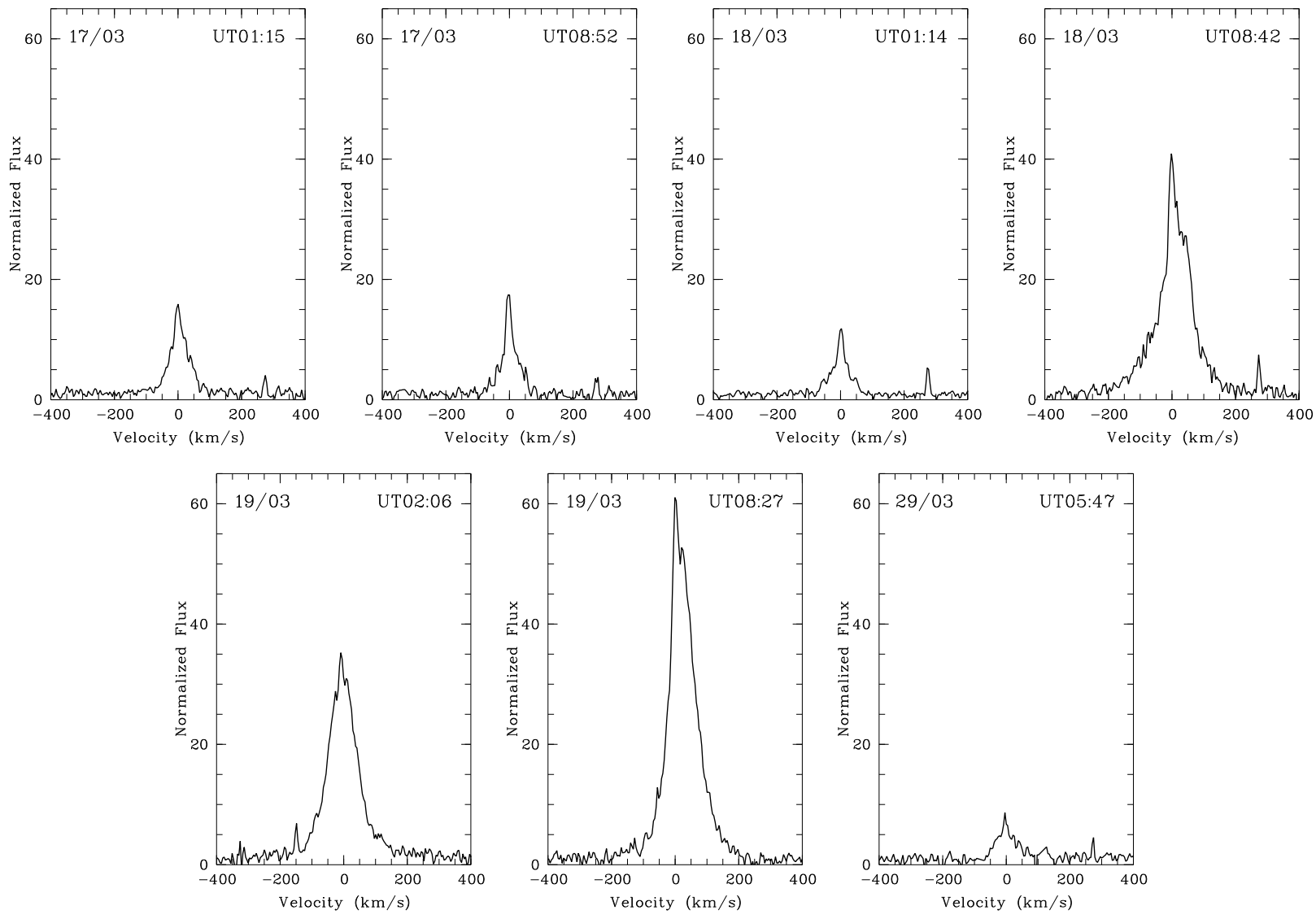

Fig. 2.- $\mathrm{H} \alpha$ line profiles for 2M1101. All fluxes are normalized to the continuum. Emission features at $\sim 270 \mathrm{kms}^{-1}$ have their origin in non-perfect background subtraction. 

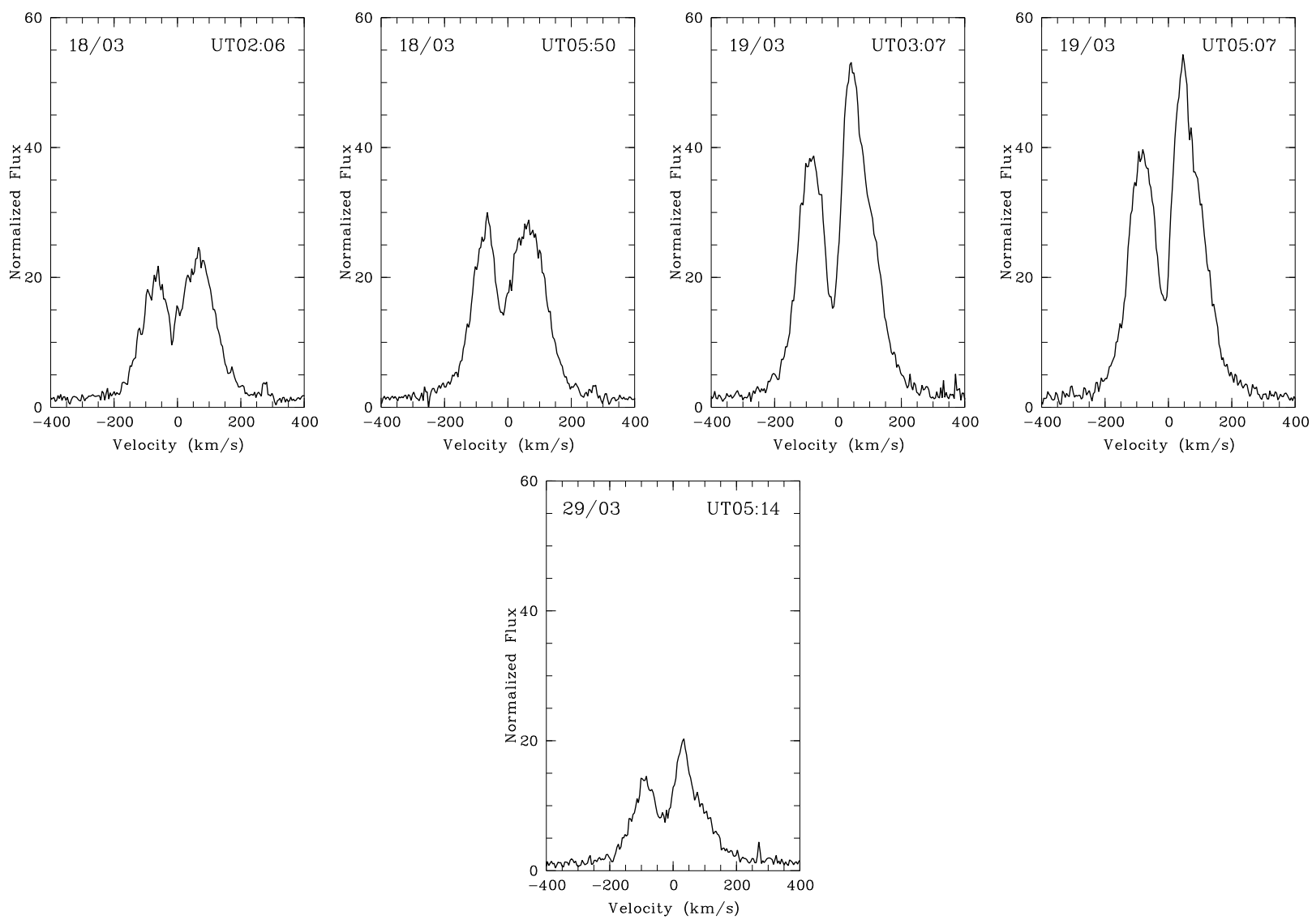

Fig. 3.- H $\alpha$ line profiles for ISO217. All fluxes are normalized to the continuum. Emission features at $\sim 270 \mathrm{kms}^{-1}$ have their origin in non-perfect background subtraction. 

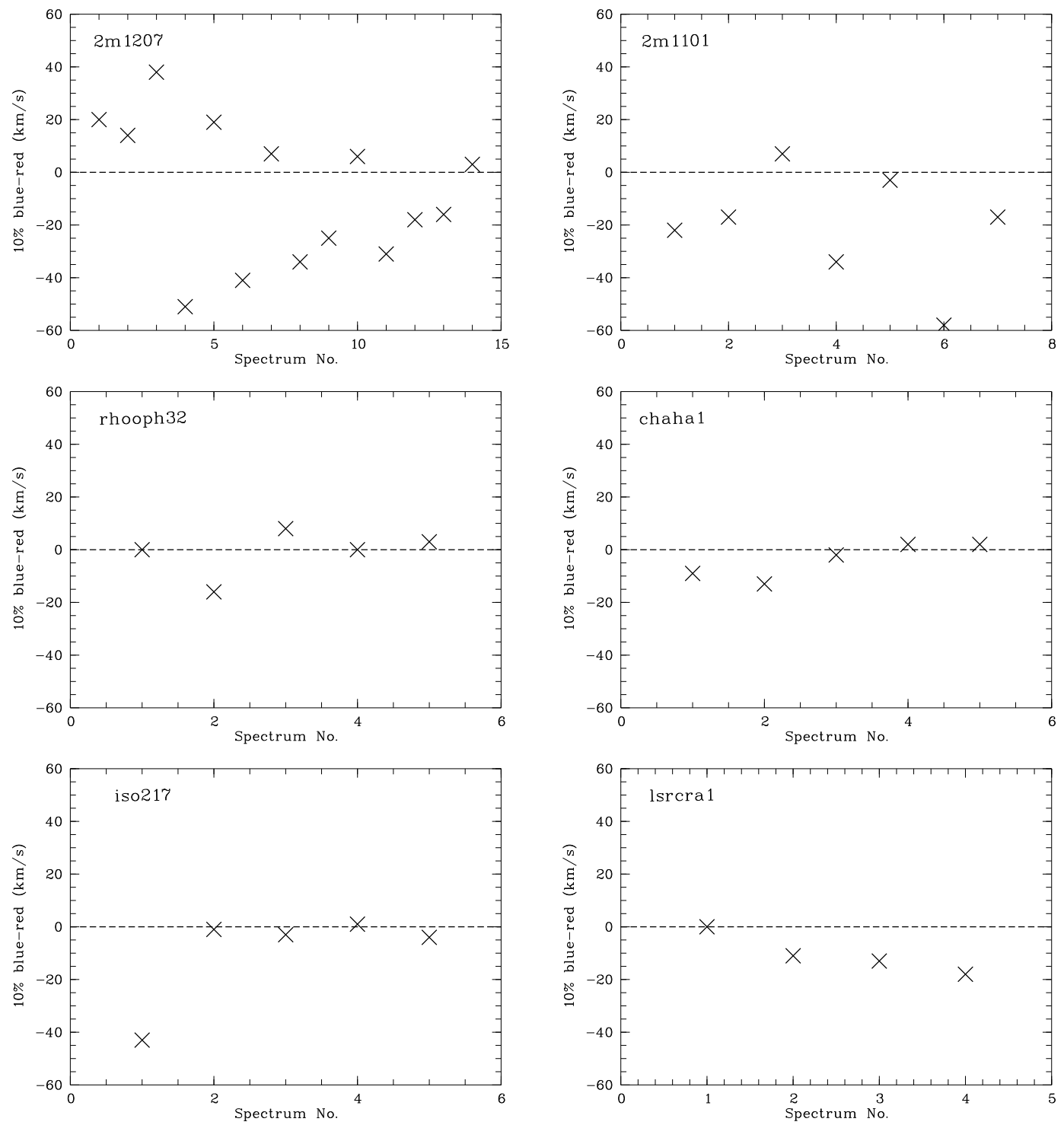

Fig. 4.- Asymmetry in the $\mathrm{H} \alpha$ profile, measured as the difference between blue $10 \%$ width minus red $10 \%$ width. 

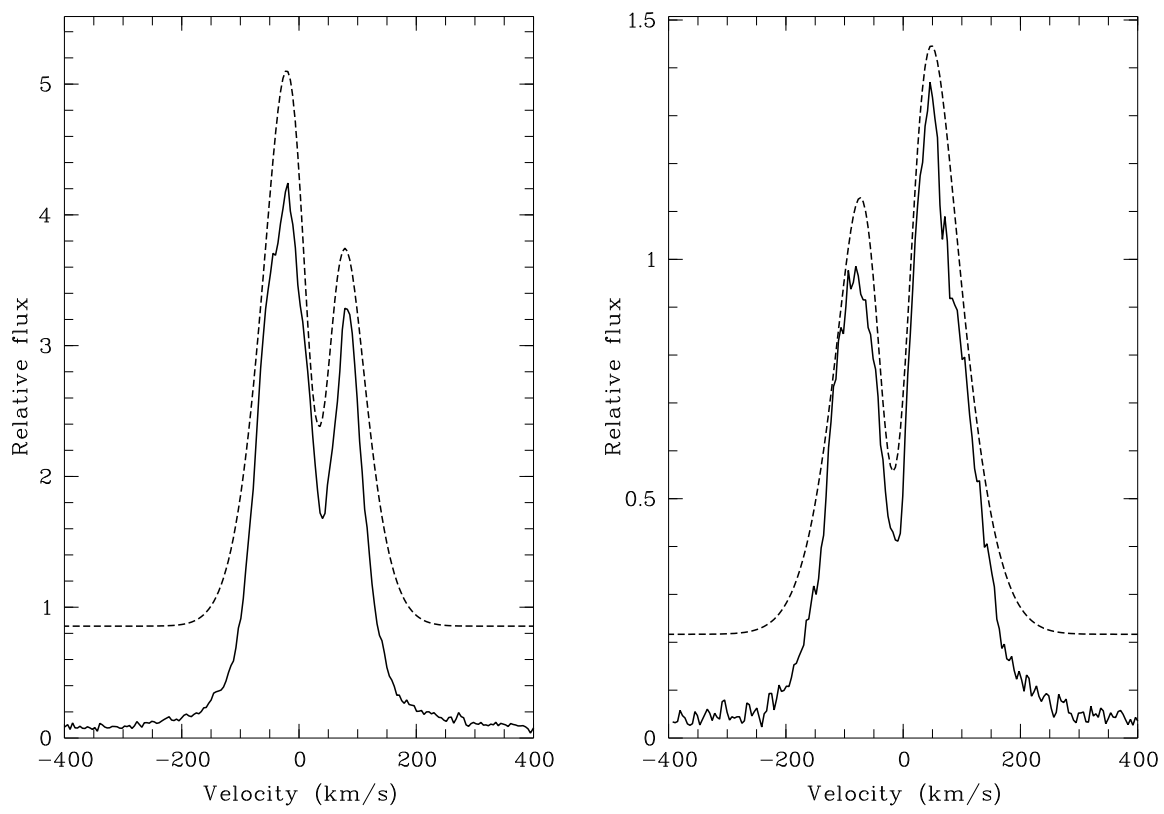

Fig. 5.- Gaussian decomposition for 2M1207 (left panel) and ISO 217 (right panel). Shown is the observed (solid) profile from an example spectrum and the fit (dashed), which is a coaddition of two Gaussians. The fit curve is shifted by 0.8 (left panel) and 0.1 (right panel) units for clarity. 